\title{
Parameterization-based tracking for the P2 experiment
}

\author{
lurii Sorokin ${ }^{1,2, a}$ for the P2 collaboration \\ ${ }^{1}$ Institute for Nuclear Physics, University of Mainz, Germany \\ ${ }^{2}$ PRISMA Cluster of Excellence
}

\begin{abstract}
The P2 experiment in Mainz aims to determine the weak mixing angle $\theta_{W}$ at low momentum transfer by measuring the parity-violating asymmetry of elastic electronproton scattering. In order to achieve the intended precision of $\Delta\left(\sin ^{2} \theta_{W}\right) / \sin ^{2} \theta_{W}=$ $0.13 \%$ within the planned 10000 hours of running the experiment has to operate at the rate of $10^{11}$ detected electrons per second. Although it is not required to measure the kinematic parameters of each individual electron, every attempt is made to achieve the highest possible throughput in the track reconstruction chain.

In the present work a parameterization-based track reconstruction method is described. It is a variation of track following, where the results of the computation-heavy steps, namely the propagation of a track to the further detector plane, and the fitting, are pre-calculated, and expressed in terms of parametric analytic functions. This makes the algorithm extremely fast, and well-suited for an implementation on an FPGA.

The method also takes implicitly into account the actual phase space distribution of the tracks already at the stage of candidate construction. Compared to a simple algorithm, that does not use such information, this allows reducing the combinatorial background by many orders of magnitude, down to $O(1)$ background candidate per one signal track.

The method is developed specifically for the P2 experiment in Mainz, and the presented implementation is tightly coupled to the experimental conditions.
\end{abstract}

\section{The P2 experiment}

\subsection{Motivation}

One of the fundamental parameters in the Standard Model is the weak mixing angle $\theta_{W}$, which defines the relative strength of the electromagnetic and weak interactions. The weak mixing angle is a scaledependent quantity and it has been measured at various energies and in different processes [1] (Fig. 1). However, the most precise measurements, performed at the $\mathrm{Z}$ pole, are marginally consistent, and the measurements at lower energies are not precise enough to discriminate between the Standard Model and possible extensions. The P2 experiment in Mainz aims at measuring the weak mixing angle at low momentum transfer (average momentum transfer squared $Q^{2}=4.5 \cdot 10^{-3} \mathrm{GeV}^{2} / \mathrm{c}^{2}$ ) to a precision of $0.13 \%$. Such measurement will be a stringent test of the Standard Model, and has the potential of discovering physics beyond the Standard Model in the running.

ae-mail: sorokin@uni-mainz.de 


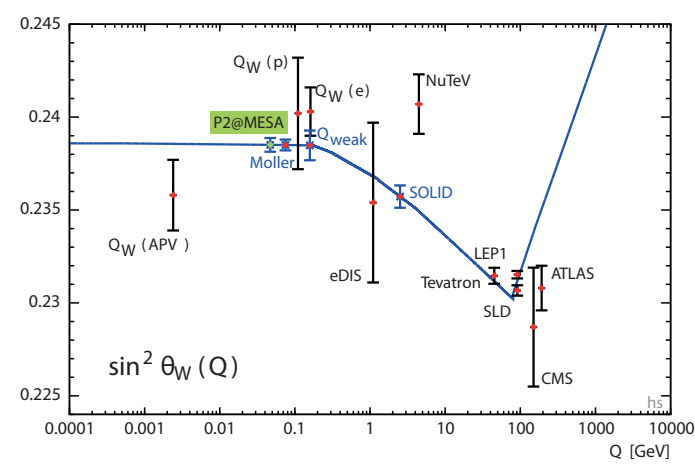

Figure 1. Scale dependence of $\sin ^{2} \theta_{W}$ together with completed (black error bars) and planned (blue error bars, value chosen to coincide with theory) experimental measurements [1].

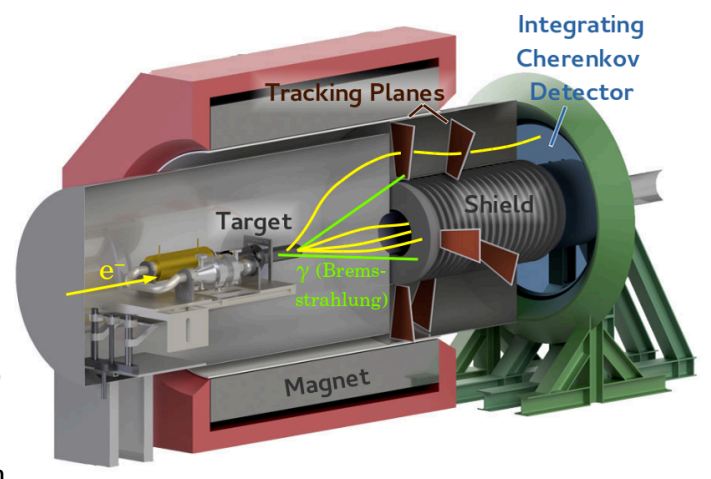

Figure 2. CAD model of the P2 detector.

\subsection{The method and the setup}

In the P2 experiment the weak mixing angle is going to be determined from the parity-violating asymmetry of elastic electron-proton scattering. The latter can be related to $\theta_{W}$ as follows:

$$
\begin{gathered}
A_{P V}=\frac{\sigma_{L}-\sigma_{R}}{\sigma_{L}+\sigma_{R}}=\frac{G_{F} Q^{2}}{4 \sqrt{2} \pi \alpha}\left(Q_{W}+F\left(Q^{2}\right)\right), \\
Q_{W}=1-4 \sin ^{2} \theta_{W},
\end{gathered}
$$

where $A_{P V}$ is the parity violating asymmetry, $\sigma_{L}$ and $\sigma_{R}$ are the elastic scattering cross-sections for left- and right-handed electrons, $Q_{W}$ is the weak charge of the proton, $F\left(Q^{2}\right)$ is the Fermi constant, $\alpha$ is the fine structure constant, and $Q^{2}$ is the momentum transfer squared.

The planned experimental setup is shown in Fig. 2. A longitudinally polarized electron beam is scattered in a liquid hydrogen target, and the current of the elastically scattered electrons is measured with a Cherenkov Detector. The beam polarization is flipped with a rate of several $\mathrm{kHz}$. Providing the integrated luminosity with both helicities is the same, the parity violating asymmetry can be evaluated as:

$$
A_{P V}=\frac{N_{L}-N_{R}}{N_{L}+N_{R}}
$$

where the $N_{L}$ and $N_{R}$ are the number of detected electrons with left-handed and right-handed helicities respectively.

As $A_{P V}$ is on the order of $10^{-8}$, to achieve the required statistical precision of $\Delta A_{P V} \leq 6 \cdot 10^{-10}$, the number of detected electrons needs to be higher than $3 \cdot 10^{18}$. In order to collect such immense statistics on a feasible timescale, assuming 10000 running hours, the experiment needs to work at a rate of $10^{11}$ detected electrons per second.

A new accelerator, MESA [2], is going to be built to provide the required high intensity $(150 \mu \mathrm{A})$, highly polarized $(>85 \%)$ beam with an exceptional stability: helicity-correlated position fluctuations below $0.13 \mathrm{~nm}$, angular fluctuations below $0.06 \mathrm{nrad}$, and intensity fluctuations below $0.36 \mathrm{ppb}$. The energy of the beam is chosen to be $155 \mathrm{MeV}$ to minimize the theoretical uncertainties [3]. 
The long $(60 \mathrm{~cm})$ target is also necessary to achieve the required event rate. A vast amount of bremsstrahlung photons is produced in the target, creating a background in the Cherenkov Detector. The lead shield in the middle of the setup blocks the photons coming directly from the target, but still a considerable amount of the photons hit the Cherenkov Detector after a Compton scattering in the inner surface of the magnet. The superconducting magnet bends the electrons around the shield.

Evaluating the weak mixing angle from equation 1 requires knowing the distribution of the momentum transfer squared $Q^{2}$. Because of the considerable energy loss and multiple scattering in the target, the $Q^{2}$ distribution need to be determined from a detailed simulation. Here one can not fully rely on GEANT4, as the precise shape of the scattering distribution is important, and helicity correlated effects are not implemented. To properly tune and validate the simulation the Tracking System is necessary.

\subsection{Tracking system}

The main purpose of the Tracking System is to help evaluating the $Q^{2}$ distribution of the primary electron-proton scattering. In addition, the Tracking System is going to be used for detector alignment, as well as for monitoring the experimental conditions by means of watching the kinematic distributions.

The $Q^{2}$ distribution needs to be determined with the relative systematic uncertainty on the mean below $0.2 \%$. The measurement will be performed at a decreased beam rate, to reduce the effect of combinatorial background. With the same data sets the detector alignment and validation of the magnetic field map will be performed.

During the normal experiment operation, at the full beam rate, the amount of data, produced by the Tracking System will be too large to be processed with the affordable computing resources. The data will be acquired in short intervals, with sufficient pauses for processing in between. Every attempt is made to achieve the highest possible throughput in the reconstruction chain, as this would result in better sensitivity to variations of the experimental conditions. The minimum required duty cycle can not be quoted at the moment, as it depends on the tracking performance and details of experimental conditions.

The Tracking System will be based on the MuPix [4] high-voltage monolithic active pixel sensors (HV-MAPS). MuPix features high rate capability (2 MHz/chip tested, $284 \mathrm{MHz} / \mathrm{cm}^{2}$ theoretical), time resolution of about $11 \mathrm{~ns}$, low mass, low power consumption $\left(300 \mathrm{~mW} / \mathrm{cm}^{2}\right)$, and high efficiency (99.5\%) [4]. The chip was originally developed for the Mu3e experiment [7], and fits very well to the needs of $\mathrm{P} 2$.

The sensors will be arranged in four planes. The choise of four planes was made to minimize the material budget. At least three planes are necessary to constrain the track parameters within a magnetic field, and at least one more is needed to distinguish between the real tracks and random hit combinations. The planes are placed in pairs, with $2 \mathrm{~cm}$ distance between the planes within each pair, and $54 \mathrm{~cm}$ between the pairs (Fig. 3). Placing the planes close to each other reduces the area to search for the matching hit, hence reduces the combinatorial background. Yet, a certain spacing is necessary to accurately measure the track curvature.

The Tracking System consists of four sectors (Fig. 2), each covering about $15^{\circ}$ of acceptance in azimuth angle $\phi$. Locating the sectors at $90^{\circ}$ and $180^{\circ}$ w.r.t. to each other is favorable to detect spatial asymmetries. 


\subsection{Reconstruction challenge}

The major challenges in track reconstruction are the extreme track rate, high hit density, and considerable background from the bremsstrahlung photons. For the first estimates it was assumed that the track reconstruction is done in $45 \mathrm{~ns}$ time frames, which corresponds approximately to four times the time resolution of the MUPIX chip (measured to be $\sigma_{t}=11 \mathrm{~ns}$ [4]). At the full beam rate this leads to about 800 reconstructible signal electrons and up to 6000 background hits per plane in one frame.

Obviously, at such high rates, the reconstruction has to be done on-line. To achieve the highest possible throughput the reconstruction is considered to be implemented on a FPGA. For this a fast and simple algorithm is required, such as the parameterization-based tracking.

\subsection{Simulations}

In the following sections references to the GEANT4 [5] simulations will be made. At present the GEANT4 model of the P2 experiment includes most of the relevant material in the detector, uses a realistic magnetic field map, and considers the relevant electromagnetic processes. The major simplifications in the model are that: (a) the incident beam is stationary, and goes along the z-axis, whereas in fact it is planned to scan with the beam over the target volume in order to prevent the target from boiling, (b) that the tracking planes are flat, without any dead area, whereas in fact they will consist of $2 \times 2 \mathrm{~cm}^{2}$ sensors, overlapping in $\mathrm{x}$ and $\mathrm{y}$ (because of the dead edges), and interleaved in $\mathrm{z}$, and (c) the misalignment and the uncertainty on the field map are not simulated. The present model is adequate for evaluating the performance of the tracking algorithm. Further extension of the model are necessary for other studies, as will become clear from the text.

\section{Parameterization-based tracking}

\subsection{Concept}

The presented parameterization-based tracking is a variation of track following. Track following is a track reconstruction method, when first track seeds are constructed, and then hits from the consequent planes are added to the seeds one-by-one. Finally, the constructed hit combinations - track candidates - are fitted, and the best candidates are selected based on the fit quality information, usually $\chi^{2}$.

In P2 the reconstruction goes backwards: each hit in plane 3 (Fig. 3) is taken as a track seed, and then hits from the planes 2, 1 and 0 are added in the respective order. A combination of one, two, or three hits, constructed in such way, will be called a track segment.

To decide which hits can be added to a track segment one needs to extrapolate the segment to the next (upstream) plane. The extrapolated position, along with the extrapolation uncertainty, define the region of interest $(\mathrm{ROI})$ - the region where all hits match to the track segment. Usually, there is more than one hit in the ROI. In such case extended segments are constructed with each of the matching hits.

Extrapolation of a segment is associated with certain difficulties. An explicit extrapolation requires an estimate for the kinematic parameters of the track segment. The kinematic parameters, in particular the momentum, can be estimated only if the segment consists of three or more hits. To find matching hits for one- and two-hit segments one would need to use a predefined, sufficiently large ROI, which is inefficient in terms of combinatorial background. In addition, the explicit extrapolation requires looking up the geometry and the magnetic field map, as well as performing numerical integration, which all together can take a significant fraction of the overall computation time. 
In the parameterization-based tracking the ROIs are pre-calculated beforehand, and expressed in terms of parametric functions of the coordinates of the segment hits. So, during the reconstruction, the optimal ROI can be easily evaluated from the parameterizations for any track segment.

In the current implementation, for the sake of simplicity, the ROIs are chosen to be rectangular. The characteristics of the rectangular ROI, namely the $x$ - and $y$-positions, the $x$ - and $y$-sizes, and the rotation are expressed as 3rd order polynomial functions of the segment hit coordinates. In the following these quantities will be referred to as $x_{\text {pos }}, y_{\text {pos }}, x_{\text {size }}, y_{\text {size }}$, and $\theta_{\text {rot }}$ respectively. How exactly $x_{\text {pos }}, y_{\text {pos }}, x_{\text {size }}, y_{\text {size }}$, and $\theta_{\text {rot }}$ depend on the coordinates of the segment hits will be explained in section 2.3. The variables $x_{\text {pos }}, y_{\text {pos }}, x_{\text {size }}, y_{\text {size }}$, and $\theta_{\text {rot }}$ express the position of the ROI respectively to the last hit in the segment, in a rotated reference frame, as shown in Fig. 4. The 3rd order polynomials were chosen also for simplicity, and planned to be replaced with splines in the future. Also, for technical reasons, in the current implementation $\theta_{\text {rot }}$ is always zero, which also needs to be fixed.

The next section (2.2) will describe how to define the ROI for a given track segment. In section 2.3 it will be shown how to proceed from a set of segments with defined ROIs to the sought parameterizations. In sections 2.4 and 3.3 it will be demonstrated that not only the ROIs, but also the track parameters, such as the position and the momentum, can be parameterized.

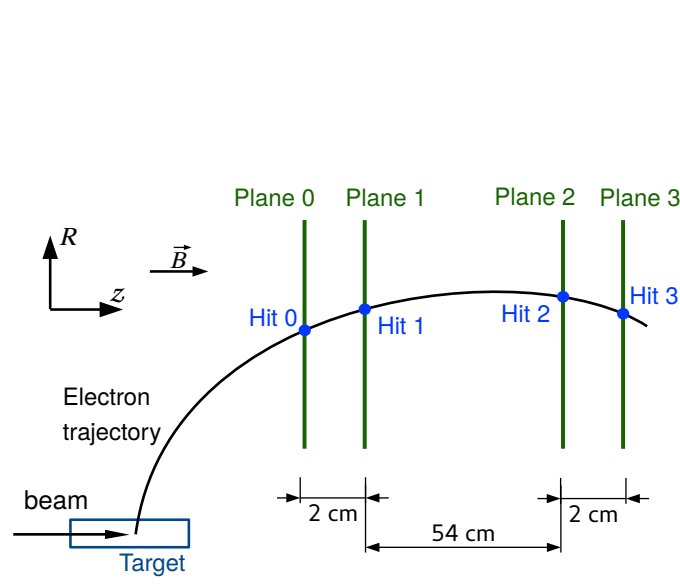

Figure 3. Schematic $z R$-view on the target and the tracking planes.

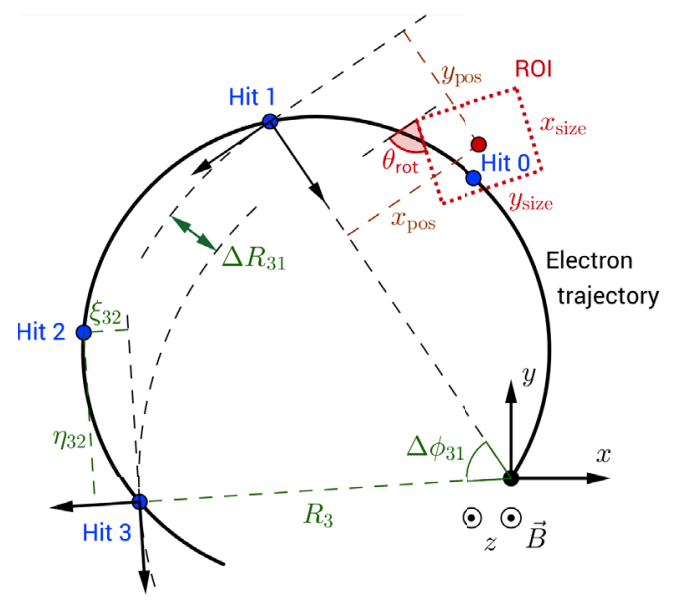

Figure 4. Schematic $x y$-view on the target and the tracking planes.

\subsection{Defining the region of interest}

In this work the ROIs are defined by comparing the segments with reference tracks. The reference tracks are either tracks from the GEANT4 simulation, or high quality (low $\chi^{2}$ ) tracks, reconstructed from the low beam rate experimental data. At low beam rate the combinatorial background is small, so track candidates can be constructed with a simple exhaustive search, and a conventional rigorous fit of every candidate can be performed.

To define the ROI one first selects reference tracks that are similar to the given track segment. More precise, it is required that each hit of the reference track would lie within a certain range around the corresponding, i.e. lying in the same plane, hit of the segment. If the segment has no hit in a certain plane, then the corresponding hit of the reference track is ignored during the selection. Having the 
reference tracks selected in this way, it is trivial to define the ROI: it is the region, where the selected reference tracks are concentrated. In the current implementation it is defined as the rectangle of the minimal area, which encloses not less than a certain fraction (typically 95\%-99\%) of the reference tracks. Obviously, the ROI, defined in such way, will enable to find only these tracks, for which similar reference tracks were available. To go beyond this limitation one can scale up the size of the obtained ROI.

\subsection{Parameterizing the region of interest}

First the general form of the parameterizations need to be defined. As mentioned in section 2.1, in the current implementation the ROIs are chosen to be rectangular, so one needs to define five functions: $x_{\text {pos }}, y_{\text {pos }}, x_{\text {size }}, y_{\text {size }}$, and $\theta_{\text {rot }}$.

The functions were chosen to be 3rd order polynomials, and must depend on the coordinates of the segment hits. But using the coordinates of all hits explicitly is disadvantageous because this would make the parameterization functions to be up to six-dimensional, which would later on lead to certain technical difficulties. The number of variables can be reduced by taking one symmetry and one correlation into account.

First the functions for the ROI in plane 2 (Fig. 3) will be defined. The ROI in plane 2 can depend only on the coordinates of the hit in plane 3 . Since the position of the ROI is defined relativeley to the last hit of the segment (hit 3), and due to the rotational symmetry of the setup, the functions $x_{\text {pos }}, y_{\text {pos }}$, $x_{\text {size }}, y_{\text {size }}$, and $\theta_{\text {rot }}$ can be defined as functions of the radial position of hit $3, R_{3}$, only (Fig. 4).

The ROI in plane 1 can depend only on hits in planes 3 and 2. Again, due to the rotational symmetry, the functions $x_{\text {pos }}, y_{\text {pos }}, x_{\text {size }}, y_{\text {size }}$, and $\theta_{\text {rot }}$ can be defined to depend only on $R_{3}$ and on the relative position of hits 2 and $3, \xi_{32}$ and $\eta_{32}$ (Fig. 4).

The ROI in plane 3 should, in general, depend on position of hits in planes 3,2, and 1 . But the positions of hits 3 and 2 become strongly correlated as soon as the position of hit 1 is fixed. Indeed, the $x$ - and $y$-position of hits 1 and 3, together with the target, make a coarse constraint on the track radius, whereas the $z$ positions of the two hits, together with the defined track radius, constrain the track inclination w.r.t. to the $x y$-plane. Even the coarse constraints on the track parameters lead to a strong correlation between hits 3 and 2 because the planes are very close to each other. Therefore, in the current implementation the position of hit 2 is ignored. Taking into account the rotational symmetry, the functions $x_{\text {pos }}, y_{\text {pos }}, x_{\text {size }}, y_{\text {size }}, \theta_{\text {rot }}$ are defined to depend on $R_{3}$, as well as on the relative position of hits 1 and $3, \Delta R_{31}$ and $\Delta \phi_{31}$ (Fig. 4). Here polar coordinates are chosen because they are less correlated.

Next step is to find the coefficients of the functions $x_{\text {pos }}, y_{\text {pos }}, x_{\text {size }}, y_{\text {size }}, \theta_{\text {rot }}$. This is done by fitting the functions to ROIs, created from the reference tracks as follows. The phase spaces of the parameterization functions, $\left\{R_{3}\right\}$ in case of plane $2,\left\{R_{3}, \xi_{32}, \eta_{32}\right\}$ in case of plane 1 , and $\left\{R_{3}, \Delta R_{31}, \Delta \phi_{31}\right\}$ in case of plane 0 , are divided in bins. All reference tracks are grouped according to each of the three binnings. For each bin in each phase space a ROI is created using the reference tracks in this bin (an example is shown in Fig. 5). The ROIs give the fit points for the functions: the center of the respective bin gives the coordinates of the point, and ROI characteristics, namely the size, the position and the rotation, give the point values. An example fit of one of the parameterization functions, $x_{\text {size }}\left(R_{3}\right)$ for the ROI in plane 2, is shown in Fig. 6.

\subsection{Parameterization of track parameters}

Similarly, to the parameterization of the ROIs, also the track parameters, such as the position and the momentum can be parameterized. It turns out that for parameterizing the momentum it is sufficient to 


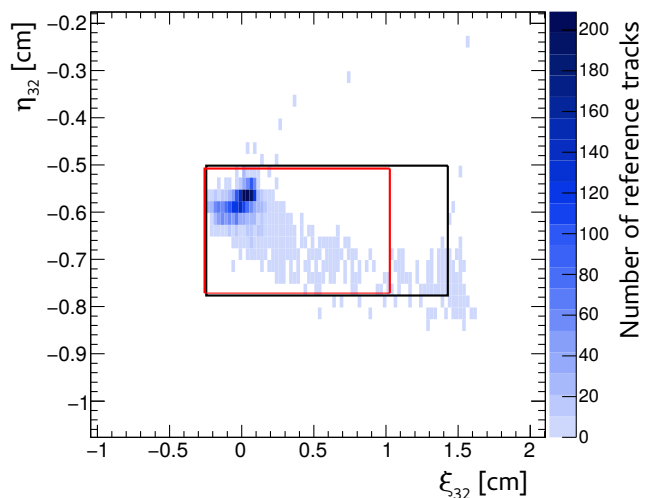

Figure 5. Spatial distribution of the reference tracks in plane 2 w.r.t. to the hit in plane 3 in the $R_{3}$ bin $[73.5 ; 74.5) \mathrm{cm}$. The coordinates are explained in Fig. 4, as well as in the text. Black rectangle is the ROI, evaluated from this spatial distribution. Red rectangle is the ROI, evaluated from the parameterizations.

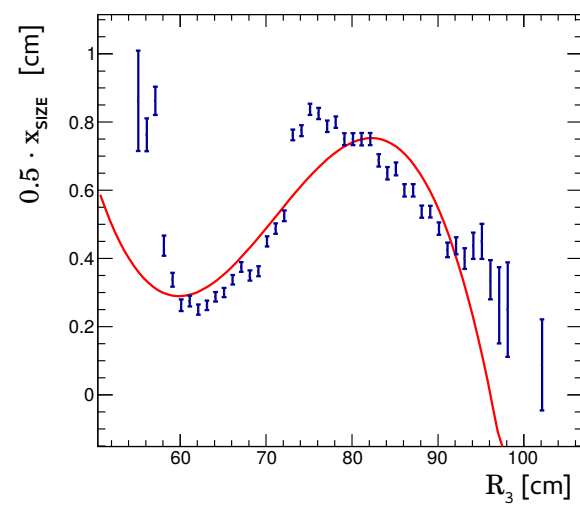

Figure 6. Fit of the $x_{\text {size }}\left(R_{3}\right)$ of the ROIs in plane 2 with a 3 rd order polynomial. The size of the bars is inversely proportional to the weight of the point, which is defined as the number of reference tracks in the bin.

consider the positions of two hits only (necessarily from different plane pairs). Indeed, the curvature of the track is additionally constrained by the position of the target, and the other two hits are strongly correlated with the selected two. For the P2 experiment so far only the parameterization of the absolute momentum was implemented. It was chosen to use the hits in planes 1 and 3 , and the variables $R_{3}, \Delta R_{31}, \Delta \phi_{31}$. The results are presented in section 3.3. However, in order to completely replace the fitting procedure one would need to parameterize the $\chi^{2}$ of fit, which necessarily requires considering the positions of all four hits. Whereas using 7 independent variables (two coordinates per hit minus one rotational symmetry) is clearly not possible in the way described above, it might be possible to either use a sophisticated function(s) of the hit coordinates as an argument for the parameterization function, or to develop (adopt) an appropriate method of evaluating the coefficients of the parameterization functions, which does not require a 7-dimensional binning. Further studies in this direction have not yet been done, but are planned.

\section{Performance tests}

A series of tests of the described tracking technique has been performed on the GEANT4-simualted data. Two different data samples were used to create the parameterizations, and to evaluate the performance. The samples were created with exactly the same GEANT4 model, as briefly described in the section 1.5.

The parameterizations were created using the reference tracks, reconstructed from simulated 45ns time frames at $0.1 \%$ of the full beam rate. All reasonable four-hit combinations were constructed and fitted with the General Broken Lines (GBL) method ([6]), and the best tracks were selected by $\chi^{2}$. 


\subsection{Efficiency of the parameterized regions of interest}

The efficiency of a parameterized ROI is defined as the ratio of the number of signal tracks, that are within the ROI, to the total number of reconstructible signal tracks. A signal track is considered reconstructible if it made a hit in each of the four tracking planes. Only electrons that hit the Cherenkov Detetor are considered as signal. A more illustrative measure of the performance of a parameterized ROI is the distribution of the position of the signal track w.r.t. the center of the ROI. Both measures of ROI performance were evaluated with the current, yet not optimized, configuration of the algorithm.

A set of reconstructible signal tracks was selected, and for each track the ROIs in planes 2, 1 and 0 were constructed. The bottom right bar chart in Fig. 7 shows the fraction of signal tracks within individual ROIs, and ROI combinations. The overall efficiency of constructing track candidates is given by the bin " 123 ", and equals $91 \%$ in the given case. The inefficiency is caused partially by imperfect parameterization with 3rd order polynomials, and partially by hard bremsstrahlung by the signal electrons.

The three histograms in Fig. 7 show the distribution of the position of the signal track w.r.t. the center of the ROI. The quantity is plotted in the ROI local reference frame (Fig. 4), and in the units of ROI half-size. In such coordinates the tracks inside $-1<x<1 \cap-1<y<1$ are within the ROI.

This test proves the concept of parameterizing the ROIs. Further studies are needed to evaluate the perfomance of this technique.
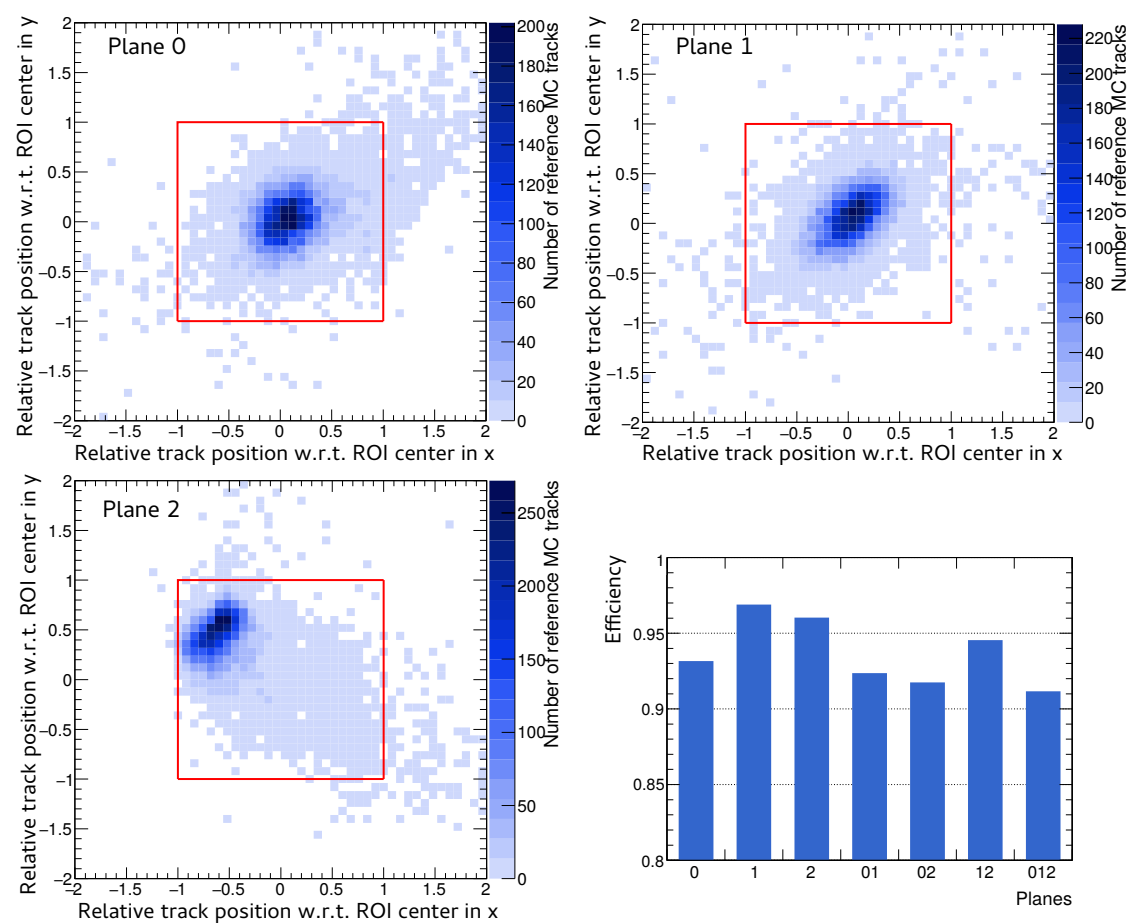

Figure 7. Top left, top right, bottom left: Position of test tracks w.r.t. to the center of the ROI, evaluated in the ROI local reference frame, and expressed in units of ROI half-size. The red squares indicate the ROI boundaries. Bottom right: efficiencies of ROI, and their combinations (explained in the text). 


\subsection{Combinatorial background}

To see whether using parameterized ROIs brings any advantages in performance, a comparison to an algorithm using constant-size ROIs was done. The constant-size ROIs were chosen to be square, their size in planes 0 and 2 was set equal, and the size was tuned to the same efficiency (91\%) as was obtained with the parameterized ROIs. The ROI in plane 1 was made so large that all hits from plane 1 were always enclosed (actually, it would be enough to select hits only from the same sector, and not from the whole plane, which would result in factor 4 fewer combinations).

Fig. 8 shows the number of constructed track candidates per one reconstructible signal track using the constant-size and the parameterized ROIs. Clearly, the difference is dramatic. One can expect that after fitting and applying a $\chi^{2}$ cut most of the wrong candidates, constructed using the fixed-size ROIs, will be rejected, and the amount of combinatorial background in the two cases will become very similar. The advantage of using parameterized ROIs is that it eliminates the need to perform the huge number of unnecessary fits.

One has to keep in mind that the parameterization-based tracking implicitly uses the information about the kinematic distribution of the true tracks, whereas the algorithm with the fixed-size ROIs doesn't.

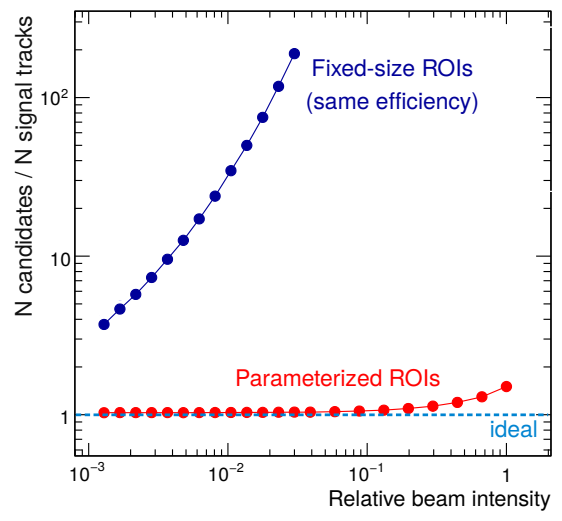

Figure 8. Number of track candidates, constructed per one reconstructible signal track, using the parameterized ROIs, and using the constant-size ROIs, tuned to the same efficiency.

\subsection{Parameterization of track momentum}

Following section 2.4 the absolute momentum was also parameterized. The accuracy of the parameterization was evaluated on the same sample of test tracks, as was used in sections 3.1 and 3.2. The obtained distribution of the relative error on the momentum is shown in Fig. 9. For comparison, the same distribution for the momentum, determined with a rigorous GBL fit is adduced. The parameterization provides a very good estimate for the track momentum. The difference in the quoted widths should rather be attributed to the imperfect Gaussian fits. The cause of the difference in the tails of the distributions is to be clarified. 

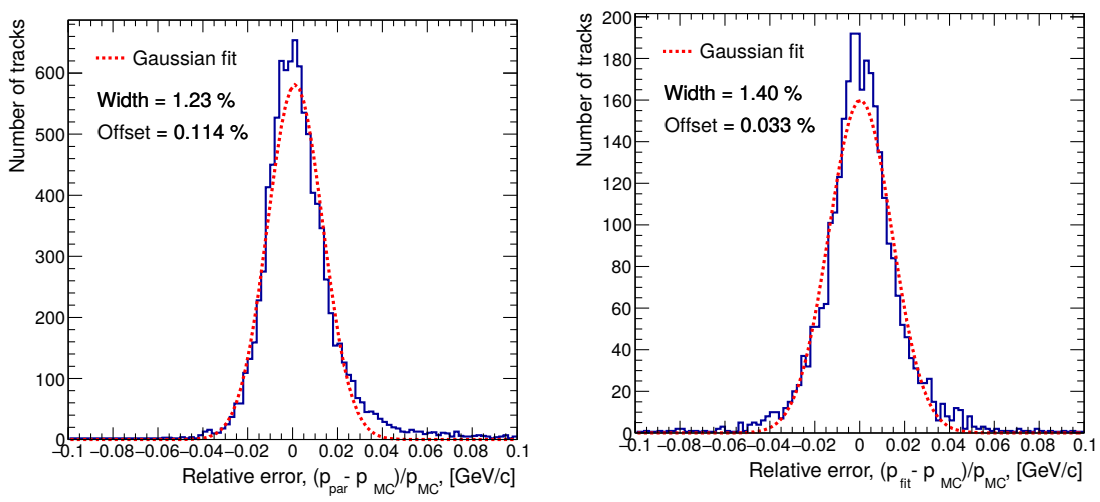

Figure 9. Relative error on the absolute momentum, evaluated from the parameterization (left), and determined with a rigorous GBL fit (right).

\section{Summary}

The parameterization-based tracking is a variation of track following, where the regions of interest are pre-calculated, and represented as a set of parametric analytic functions of the hit coordinates. In the current implementation the ROIs are defined by comparing the track segment with the reference tracks. Also the track parameters, such as the position and the momentum can be parameterized.

The parameterization-based tracking has been implemented for the P2 experiment and tested on simulated data. The first tests have proven the concept, and demonstrated a great improvement in the number of constructed track candidates, hence required fits, as compared to an algorithm with fixedsize ROIs. The parameterization of the absolute momentum is almost as accurate as the rigorous fit.

\section{References}

[1] N. Berger et al., J. Univ. Sci. Tech. China 46, no.6, 481-487 (2016)

[2] K. Aulenbacher, AIP Conf. Proc. 1563, 5-12 (2013).

[3] R. Bucoveanu, M. Gorchtein, H. Spiesberger PoS LL2016, 061, (2016)

[4] H. Augustin et al., Nucl.Instrum.Meth. A845, 194-198 (2017)

[5] J. Allison et al. Nucl.Instrum.Meth. A835, 186-225 (2016)

[6] C. Kleinwort, Nucl.Instrum.Meth. A673 107-110 (2012)

[7] A.-K. Perrevoort for the Mu3e collaboration EPJ Web Conf. 1181028 (2016)

[8] H. Augustin et al., JINST 12, no.01, C01087 (2017) 